\begin{tabular}{|c|c|c|c|c|c|c|c|c|}
\hline Reason for referral & Anaemia & Abdominal pain & Abnormal LFTs & Weight loss & $\begin{array}{l}\text { Vomiting } \\
\text { or Dysphagia }\end{array}$ & Abnormal tests or imaging & Change in bowel habit & PR bleeding \\
\hline TWR $(\%)$ & 17.3 & 9.3 & 3.8 & 19.2 & 26.9 & 7.7 & 7.7 & 7.7 \\
\hline Non-TWR (\%) & 6.7 & 33.7 & 10.1 & 0.0 & 15.7 & 14.6 & 14.6 & 4.5 \\
\hline
\end{tabular}

male 36.0\%). Reason for referral for TWR/non-TWR is shown in the table below:

$76.9 \%$ of TWR patients had an endoscopic procedure compared to $62.9 \%$ of non TWR patients $(\mathrm{p}=0.09)$. A similar percentage of patients in both groups underwent radiological investigation (TWR: 53.85\%, non-TWR: 50.56\%). More TWR patients underwent second imaging than non-TWR $(9.6 \%$ vs. 6.7). $7.7 \%$ of TWR patients and 3.4\% of non TWR patients had an end diagnosis of cancer, although this difference did not reach statistical significance. The mean age of the cancer patients in the TWR and non-TWR group was 70.3 years and 66.3 years respectively (although 2 of these in the non-TWR were below 65). 23.1 and $19.1 \%$ of patients had no clear diagnosis at 3 months in the TWR and non-TWR respectively. The mean cost of investigations and follow-up was significantly higher in the TWR cohort ( $£ 754.1$ vs $£ 613.1, \mathrm{p}=0.04$ ).

Conclusion In our sample of patients, those referred under the TWR pathway underwent a higher burden of invasive investigation with no significant increase in cancer pick up, despite being significantly more costly. The current system possibly delays cancer diagnoses in younger patients, who are more likely to be filtered through the non-TWR pathway. Perhaps alternative referral pathways need to be considered in a bid to improve cancer diagnosis in high risk patients.

Disclosure of Interest None Declared.

\section{PTH-018 INTRAVENOUS FERRIC CARBOXYMALTOSE (FERINJECT) GIVEN TO PATIENTS POST ENDOSCOPY - SAFE AND COST EFFECTIVE}

AM Verma*, D Das, A Dixon, AP Chilton. Gastroenterology, Kettering General Hospital NHS Foundation Trust, Kettering, UK

\subsection{6/gutjpl-2014-307263.464}

Introduction Intravenous (IV) iron is established as an effective management for patients with iron deficiency anaemia (IDA) and is indicated in those with severe iron deficiency, malabsorption, intolerance to oral iron and/or those requiring rapid correction of IDA. ${ }^{1}$

The use of traditional agents iron dextran (CosmoFer) and iron sucrose (Venofer) are inexpensive in drug cost (£50-60), however they require test doses, slow intravenous infusions $(4 \mathrm{~h}$ $+)$ and the incidence of hypersensitivity reactions are high. This required a day case attendance which increased the overall cost of an infusion significantly.

Then introduction of Ferric Carboxymaltose (FCM) has changed the landscape. Whilst the drug cost is 4 times higher, infusions only take $15 \mathrm{~min}$ and are much better tolerated. Also a higher dose of $1 \mathrm{~g}$ can be given. This only requires a short hospital appointment and reduces the overall cost of an infusion to less than traditional agents.

The majority of patients with IDA undergo endoscopy for investigation +/- management. We trialled the use of IV FCM in patients whom it is indicated during the post-procedure observational time.
Methods Since January 2013, patients receiving IV FCM post endoscopy had their details recorded in a database.

Results - 15 patients underwent IV FCM infusion post endoscopy;

- 9 women / 6 men, age range 33-83, mean age 64/ median 70

- Mean dose 940mg / median 935.7mg (12 patients received 1g IV);

- 13 patients received IV sedation during endoscopy;

- 6 had gastroscopy, 9 had colonoscopy;

- Indication (s): Intolerant to oral iron 8, severe IDA 9 (GAVE, CRC), malabsorption 1;

- Adverse events: 1 patient with bruising post IV cannula removal (not related to FCM).

Conclusion The safety and cost effectiveness of IV FCM has been established. This study shows that this is also the case for patients who receive IV FCM post endoscopy.

The benefits of this approach are significant. Patients are already observed post-endoscopy so giving a short IV infusion is appropriate and does not require extra staff. This is also very convenient for patients, reducing the number of attendances required in addition to the benefits of IV iron for anaemia. Giving IV FCM post endoscopy reduces the costs significantly when compared those of a separate appointment to a day case unit.

\section{REFERENCE}

1 Andrew F Goddard, Martin W James, Alistair S McIntyre, Brian B Scott, on behalf of the British Society of Gastroenterology. Guidelines for the Management of Iron Deficiency Anaemia. Gut 2011:60:1309-1316

Disclosure of Interest None Declared.

\section{PTH-019 THE EFFECT OF THREE INTERVENTIONS ON COLONOSCOPY QUALITY OUTCOMES - AN EXEMPLAR FOR ENDOSCOPY UNITS}

AM Verma*, A Dixon, AP Chilton. Gastroenterology, Kettering General Hospital NHS Foundation Trust, Kettering, UK

\subsection{6/gutjnl-2014-307263.465}

Introduction Kettering General Hospital's endoscopy unit performs $>1300$ colonoscopies per annum (symptomatic, surveillance and bowel cancer screening).

During 2012, three interventions took place in the unit.

1. The vetting guidelines for requesting colonoscopy were updated to reflect BSG guidance for the management of iron deficiency anaemia and NICE guidelines for colorectal cancer. The aim was to reduce the number of inappropriate colonoscopies, especially in those patients not fit for colonoscopy.

2. The information leaflets sent to patients pre-colonoscopy had a prompt added to urge patients to take the full dose of 4 sachets of Klean Prep (polyethylene glycol) to improve the diagnostic quality and exclusion value.

3. Three colonoscopists who were not meeting key performance indicators stopped performing colonoscopy. This allowed the remaining operators to increase the number of colonoscopies they perform. 